\title{
On Persistence and Stability of some Biological Systems with Cross Diffusion
}

\author{
E. Ahmed ${ }^{1,2}$, A. S. Hegazi ${ }^{1}$ and A. S. Elgazzar ${ }^{3,4}$ \\ 1. Mathematics Department, Faculty of Science \\ 35516 Mansoura, Egypt \\ 2. Mathematics Department, Faculty of Science \\ Al-Ain, P. O. Box 17551, UAE \\ ${ }^{3}$ Mathematics Department, Faculty of Science - Al-Jouf \\ King Soad University, Kingdom of Saudi Arabia \\ 4. Mathematics Department, Faculty of Education \\ 45111 El-Arish, Egypt
}

November 10, 2018

\begin{abstract}
The concept of cross diffusion is applied to some biological systems. The conditions for persistence and Turing instability in the presence of cross diffusion are derived. Many examples including: predatorprey, epidemics (with and without delay), hawk-dove-retaliate and prisoner's dilemma games are given.
\end{abstract}

\section{Introduction}

Cross diffusion is the diffusion of one type of species due to the presence of another [Okubo 1980, Chattopadhyay and Chatterjee 2001]. This phenomena is abundant in nature e.g. predator-prey systems where the predator diffuses towards the regions where the prey is more abundant. On the other hand, the prey tries to avoid predators by diffusing away from them. Another area of 
application is in epidemics where susceptible individuals try to avoid infected individuals.

Our aim is to generalize some biological systems to the case of the presence of cross diffusion. We study the persistence and Turing instability of the generalized models. Several examples are given e.g. predator-prey system, epidemic model, hawk-dove-retaliate game and prisoner's dilemma game.

The paper is organized as follows: In section 2 the conditions for Turing instability in the presence of cross diffusion are derived then applied to a predator-prey system. Necessary conditions for persistence of a predator prey system in the presence of cross diffusion are derived. In section 3, cross diffusion is introduced in an epidemic model. Its effect on wave solution with and without delay is derived. In section 4, the effect of cross diffusion on evolutionarily stable strategy is studied. Hawk-dove-retaliate game is given as an example. Cross diffusion is applied to the dynamics of learning in multiagent systems in section 5. Some conclusions are summarized in section 6 .

\section{Cross diffusion in a predator-prey model}

Cross diffusion is expected to be relevant to predator-prey systems where the predator diffuses towards the regions where the prey is more abundant. On the other hand, the prey tries to avoid predators by diffusing away from them. Cross diffusion term expresses the population flux of one species due to the presence of another species. The value of the cross diffusion may be positive, negative or zero.

Positive cross diffusion means that one type of species tends to move in the direction of lower density of the other type and vice versa. Hence the cross diffusion term is positive for the prey and negative for the predator. Generalizing Lotka-Volterra predator-prey model (with prey saturation) to include cross diffusion, one gets

$$
\begin{aligned}
& \frac{\partial u_{1}}{\partial t}=u_{1}\left(\alpha_{1}-\beta_{1} u_{1}-u_{2}\right)+D_{1} \frac{\partial^{2} u_{1}}{\partial x^{2}}+\frac{\partial}{\partial x}\left(D_{12}^{\prime}\left(u_{1}\right) \frac{\partial u_{2}}{\partial x}\right), \\
& \frac{\partial u_{2}}{\partial t}=u_{2}\left(-\alpha_{2}+u_{1}\right)+D_{2} \frac{\partial^{2} u_{2}}{\partial x^{2}}-\frac{\partial}{\partial x}\left(D_{21}^{\prime}\left(u_{2}\right) \frac{\partial u_{1}}{\partial x}\right),
\end{aligned}
$$

where $D_{12}^{\prime}\left(u_{1}\right)$ is the density dependent diffusion coefficient of cross diffusion such that $D_{12}^{\prime}\left(u_{1}\right) \rightarrow 0$ as $u_{1} \rightarrow 0$. Following Chattopadhyay and Chatterjee [Chattopadhyay and Chatterjee 2001] we assume that

$$
D_{12}^{\prime}=D_{12} u_{1} /\left(\epsilon+u_{1}\right) \approx D_{12}\left(1-\epsilon / u_{1}+\ldots\right),
$$


where $\epsilon$ is very small hence

$$
D_{12}^{\prime} \approx D_{12} \forall u_{1}>>\epsilon
$$

Similarly for $D_{21}$ thus we get

$$
\begin{aligned}
& \frac{\partial u_{1}}{\partial t}=u_{1}\left(\alpha_{1}-\beta_{1} u_{1}-u_{2}\right)+D_{1} \frac{\partial^{2} u_{1}}{\partial x^{2}}+D_{12} \frac{\partial^{2} u_{2}}{\partial x^{2}}, \\
& \frac{\partial u_{2}}{\partial t}=u_{2}\left(-\alpha_{2}+u_{1}\right)+D_{2} \frac{\partial^{2} u_{2}}{\partial x^{2}}-D_{21} \frac{\partial^{2} u_{1}}{\partial x^{2}},
\end{aligned}
$$

where $u_{1}\left(u_{2}\right)$ is the prey (predator) density, and all the constants $\alpha_{1}, \alpha_{2}, \beta_{1}$, $D_{1}, D_{2}, D_{12}, D_{21}$ are nonnegative. We have used $-D_{21}$ since predators move to regions with higher prey density. The value $\alpha_{1}$ is the net growth rate of the prey population in the absence of predators, while $\alpha_{2}$ is the net death rate of predators due to the absence of prey. The term $\beta_{1} u_{1}$ corresponds to the competition within the prey species.

The existence of solutions of systems similar to (2) has been studied in [Chen et al 2003 and Le 2003].

This system has a unique homogeneous coexistence solution

$$
u_{1}^{*}=\alpha_{2}, \quad u_{2}^{*}=\alpha_{1}-\alpha_{2} \beta_{1}, \quad \alpha_{1}-\alpha_{2} \beta_{1}>0 .
$$

It is straightforward to see that the solution (3) is asymptotically stable in the case of no diffusion $\left(D_{1}=0, D_{2}=0, D_{21}=0, D_{12}=0\right)$.

In Turing instability [Okubo 1980], it is required to see whether diffusion terms can destabilize the steady state (2). We derive Turing instability in the presence of cross diffusion: Consider two interacting species with different diffusion coefficients

$$
\begin{aligned}
\frac{\partial u_{1}}{\partial t} & =f_{1}\left(u_{1}, u_{2}\right)+D_{1} \frac{\partial^{2} u_{1}}{\partial x^{2}}+D_{12} \frac{\partial^{2} u_{2}}{\partial x^{2}} \\
\frac{\partial u_{2}}{\partial t} & =f_{2}\left(u_{1}, u_{2}\right)+D_{2} \frac{\partial^{2} u_{2}}{\partial x^{2}}+D_{21} \frac{\partial^{2} u_{1}}{\partial x^{2}}
\end{aligned}
$$

Assume that $\left(u_{1}^{*}, u_{2}^{*}\right)$ are the spatially uniform steady states i.e. $f_{j}\left(u_{1}^{*}, u_{2}^{*}\right)=$ $0, j=1,2$. To examine the linear stability of $\left(u_{1}^{*}, u_{2}^{*}\right)$ let

$$
u_{j}=u_{j}^{*}+\varepsilon_{j} \exp (i k x+\lambda t), \quad j=1,2 .
$$

One gets

$$
\left|\begin{array}{cc}
\lambda+D_{1} k^{2}-a_{11} & -a_{12}+D_{12} k^{2} \\
-a_{21}+D_{21} k^{2} & \lambda+D_{2} k^{2}-a_{22}
\end{array}\right|=0
$$


where

$$
\begin{aligned}
\lambda & =\frac{1}{2}\left[\left(b_{11}+b_{22}\right) \pm \sqrt{\left(b_{11}+b_{22}\right)^{2}-4\left(b_{11} b_{22}-b_{12} b_{21}\right)}\right] \\
b_{j j} & =a_{j j}-D_{j} k^{2}, \quad b_{i j}=a_{i j}-D_{i j} k^{2}, i \neq j, \\
a_{i j} & =\frac{\partial f_{i}\left(u_{1}^{*}, u_{2}^{*}\right)}{\partial u_{j}} .
\end{aligned}
$$

Turing instability occurs when $\left(u_{1}^{*}, u_{2}^{*}\right)$ is asymptotically stable if diffusion does not exist $(k=0)$; but unstable if diffusion exists $(k \neq 0)$. Thus the conditions for Turing instability are

$$
a_{11}+a_{22}<0, \quad a_{11} a_{22}-a_{12} a_{21}>0, b_{11} b_{22}-b_{12} b_{21}<0 .
$$

The third condition implies

$$
\begin{aligned}
& \left(D_{1} D_{2}-D_{12} D_{21}\right) k^{4}-\left(D_{1} a_{22}+D_{2} a_{11}-D_{12} a_{21}-D_{21} a_{12}\right) k^{2}+ \\
& \left(a_{11} a_{22}-a_{12} a_{21}\right)<0, \quad \forall k .
\end{aligned}
$$

Minimizing the left hand side of the above inequality with respect to $k$ one gets the conditions for Turing instability in the following form

$$
\left(D_{1} a_{22}+D_{2} a_{11}-D_{12} a_{21}-D_{21} a_{12}\right)>2 \sqrt{\left(a_{11} a_{22}-a_{12} a_{21}\right)\left(D_{1} D_{2}-D_{12} D_{21}\right)}>0 .
$$

Now we consider Turing instability for the system (2). Following the above procedure one gets:

Proposition 1: The system (2) is Turing stable if

$$
D_{21}>0, \quad D_{12}>0
$$

Proof: For the system (2), we have

$$
a_{11}=-\beta_{1} \alpha_{2}<0, \quad a_{12}=-\alpha_{2}<0, \quad a_{21}=\alpha_{1}-\beta_{1} \alpha_{2}>0, \quad a_{22}=0 .
$$

Since $D_{1} \geq 0, D_{2} \geq 0$, then

$$
\left(D_{1} a_{22}+D_{2} a_{11}-D_{12} a_{21}-D_{21} a_{12}\right)<0,
$$


hence the condition (6) is not satisfied.

Now the persistence of the system (2) is discussed:

Definition 1: A partial differential equation

$$
F(t, x, u(t, x), \partial u / \partial t, \partial u / \partial x, \ldots)=0
$$

defined on a spatial domain $\Omega$ is persistent in the context of zero Dirichlet boundary conditions if there exist a region $V=\{u: 0<u<w\}$ in $\Omega$ such that all solutions with nontrivial, nonnegative initial conditions are attracted to $V$.

Equivalently the boundary of the positive cone in the space where the solutions exist act as a repeller.

Persistence [Hofbauer and Sigmund 1998] is an important concept in population biology since it means that a certain species will continue to exist. The work of Cantrell et al [Cantrell et al 1993] is basic in finding sufficient conditions for persistence in some partial differential equations without cross diffusion. Therefore we will use it to derive the necessary conditions for persistence of the system (2). Here zero Dirichlit boundary conditions are assumed:

$$
u_{i}=0 \text { at } x=0, x=L .
$$

For the sake of completeness we mention the following results [Cantrell et al 1993] with slight modifications:

\section{Theorem (1):}

(i) Suppose that $f(\vec{r}, u)$ is Lipschitz in $\vec{r}$ and continuously differentiable in $u$ with

$$
\partial f / \partial u \leq 0 \text { for } u \geq 0, f(\vec{r}, u) \leq 0 \text { if } u \geq l
$$

for some constant $l$, and $f(\vec{r}, 0)>0$ at some point in the domain $\Omega$, then the problem

$$
\partial u / \partial t=D \nabla^{2} u+u f(\vec{r}, u) \quad \text { in } \Omega \times(0 . \infty),
$$


with Dirichlet or Neumann boundary conditions has a unique positive steady state $u^{s s}$ which is a global attractor for nontrivial non-negative solutions (hence the system (8) is persistent) if the following problem has a positive eigenvalue $\sigma$

$$
\sigma u=D \nabla^{2} u+u f(\vec{r}, 0) \quad \text { in } \Omega,
$$

with the same boundary conditions as (8).

(ii) Suppose that $f_{1}\left(\vec{r}, u_{1}, u_{2}\right)$ and $f_{2}\left(\vec{r}, u_{1}, u_{2}\right)$ are $C^{2}$,bounded, that $f_{1}\left(\vec{r}, u_{1}, 0\right)$ satisfies the conditions of part (i) with positive $\sigma$, that $f_{2}\left(\vec{r}, 0, u_{2}\right) \leq 0$ for $u_{2} \geq 0$. Let $\sigma_{0}$ be the largest eigenvalue of the system

$$
\sigma_{0} u_{1}=D_{1} \nabla^{2} u_{1}+u_{1} f_{1}(\vec{r}, 0,0) \quad \text { in } \Omega
$$

with Dirichlet or Neumann boundary conditions. Also let $\sigma_{2}$ be the largest eigenvalue of the system

$$
\sigma_{2} u_{2}=D_{2} \nabla^{2} u_{2}+u_{2} f_{2}\left(\vec{r}, u_{1}^{s s}, 0\right) \quad \text { in } \Omega
$$

where $u_{1}^{s s}$ is given by $f_{1}\left(\vec{r}, u_{1}^{s s}, 0\right)=0$, then the following system is persistent if both $\sigma_{0}$ and $\sigma_{2}$ are positive

$$
\begin{aligned}
& \partial u_{1} / \partial t=D_{1} \nabla^{2} u_{1}+u_{1} f_{1}\left(\vec{r}, u_{1}, u_{2}\right) \\
& \partial u_{2} / \partial t=D_{2} \nabla^{2} u_{2}+u_{2} f_{2}\left(\vec{r}, u_{1}, u_{2}\right) \quad \text { in } \Omega \times(0 . \infty) .
\end{aligned}
$$

Now we consider the persistence of system (2). The first persistence criteria is that the prey model alone (without predators) should be persistent, hence [Cantrell et al 1993]

$$
\alpha_{1}>D_{1}\left(\frac{\pi}{L}\right)^{2}
$$

The second criteria is that the predator system should be persistent when the prey is at its maximum capacity $u_{1}=\alpha_{1} / \beta_{1}$, hence

$$
\frac{\alpha_{1}}{\beta_{1}}-\alpha_{2}>D_{2}\left(\frac{\pi}{L}\right)^{2} .
$$

Now we study persistence in the presence of cross diffusion. The most critical region is where both $u_{1}$ and $u_{2}$ are small, hence we assume that 


$$
u_{j}=\varepsilon_{j} \exp (\lambda t) \sin \left(\frac{\pi x}{L}\right), \quad j=1,2,
$$

and linearize in $\varepsilon_{j}$, we get the following sufficient conditions for persistence $(\lambda>0)$.

Proposition 2: In addition to Eqs. (9) and (10) one of the following conditions is necessary for the persistence of the system (2):

$$
\alpha_{1}-\alpha_{2}>\left(D_{1}+D_{2}\right)\left(\frac{\pi}{L}\right)^{2}
$$

or

$$
\left[\alpha_{1}-D_{1}\left(\frac{\pi}{L}\right)^{2}\right]\left[\alpha_{2}+D_{2}\left(\frac{\pi}{L}\right)^{2}\right]>D_{12} D_{21}\left(\frac{\pi}{L}\right)^{4} .
$$

Proof: Substituting $u_{j}$ in $(2)$ and linearizing in $\varepsilon_{j}$ one gets

$$
\begin{aligned}
& \varepsilon_{1}\left[\lambda-\alpha_{1}+D_{1}(\pi / L)^{2}\right]+\varepsilon_{2} D_{12}(\pi / L)^{2}=0 \\
& \varepsilon_{2}\left[\lambda+\alpha_{2}+D_{2}(\pi / L)^{2}\right]-\varepsilon_{1} D_{21}(\pi / L)^{2}=0
\end{aligned}
$$

To get a nonzero solution the determinant of the coefficients has to be zero which gives the following equation

$$
\begin{aligned}
& \lambda^{2}+\lambda\left[-\left(\alpha_{1}-\alpha_{2}\right)+\left(D_{1}+D_{2}\right)\left(\frac{\pi}{L}\right)^{2}\right]+\left[\left(-\alpha_{1}+D_{1}\left(\frac{\pi}{L}\right)^{2}\right)\left(\alpha_{2}+D_{2}\left(\frac{\pi}{L}\right)^{2}\right)+\right. \\
& D_{12} D_{21}\left(\frac{\pi}{L}\right)^{4}=0
\end{aligned}
$$

Persistence requires that the real part of one of the roots, at least, should be positive hence

$$
\alpha_{1}-\alpha_{2}>\left(D_{1}+D_{2}\right)\left(\frac{\pi}{L}\right)^{2}
$$

or

$$
\left[\alpha_{1}-D_{1}\left(\frac{\pi}{L}\right)^{2}\right]\left[\alpha_{2}+D_{2}\left(\frac{\pi}{L}\right)^{2}\right]>D_{12} D_{21}\left(\frac{\pi}{L}\right)^{4} .
$$

This completes the proof. 


\section{Cross diffusion in epidemics}

In this section, we will study an epidemic model where the population is classified into susceptible (S), and infected and infective (I), according to the health of each individual. The transition between the two states occurs according to the following rules:

Susceptible individual having at least one infected nearest neighbor is infected in the next time step.

Infected individuals are recovered and become susceptible with a rate $\lambda$.

Cross diffusion will be included, where S-individuals diffuse away from I-individuals. This system will be studied both without and with delay. In the first case we have

$$
\begin{aligned}
& \frac{\partial S}{\partial t}=-S I, \\
& \frac{\partial I}{\partial t}=S I-\lambda I-D \frac{\partial^{2} S}{\partial x^{2}},
\end{aligned}
$$

where both $\lambda$ and $D$ are nonnegative constants.

Here we consider that only susceptibles are capable of diffusing. This corresponds to the case where the disease has symptoms hence susceptibles try to avoid infectives.

Studying the wave solution of Eq. (14)

$$
S=S(z), I=I(z), z=x-c t
$$

with the boundary conditions

$$
S(\infty) \simeq 1,0 \leq S(-\infty) \leq 1, I(\infty)=I(-\infty) \simeq 0
$$

one gets

$$
I=c \frac{S^{\prime}}{S},-c I^{\prime}=-D S^{\prime \prime}-\lambda I+S I, \quad S^{\prime}=\frac{\mathrm{d} S}{\mathrm{~d} z}, I^{\prime}=\frac{\mathrm{d} I}{\mathrm{~d} z} .
$$

Substituting from the first equation and integrating the second one, we get

$$
F-c I=-D \frac{S I}{c}-\lambda c \ln S+c S \text {. }
$$


To determine the constant $(F)$, consider $z \rightarrow \infty$ i.e. the past then Eq. (15) implies that $F=c$. Now consider the future $z \rightarrow-\infty$, and let $S(t=\infty)=\sigma$, then the fraction of survivors $\sigma$ is given by the transcendental equation as follows:

$$
\frac{1-\sigma}{\ln (\sigma)}=\lambda
$$

which is identical to an equation obtained by Murray [Murray 1993] in another context.

It is straightforward to see that the homogeneous solution $(S=1, I=0)$ is asymptotically stable if

$$
\lambda<1, \quad c \geq \sqrt{D} .
$$

Introducing delay, the system (14) becomes

$$
\begin{aligned}
& \frac{\partial S}{\partial t}=-S I \\
& \frac{\partial I}{\partial t}=S(x, t-T) I(x, t-T)-\lambda I-D \frac{\partial^{2} S}{\partial x^{2}} .
\end{aligned}
$$

This model is similar but not identical to that of Mendez [Mendez 1998]. Consider the wave solution of Eq. (18) with the boundary conditions (15), and studying the stability of the homogeneous solution $(S=1, I=0)$, then one gets

$$
\left(c-\frac{D}{c}\right) I^{\prime}-\lambda I+\sum_{n=0} \frac{(c T)^{n}}{n !} \frac{\mathrm{d}^{n} I}{\mathrm{~d} z^{n}}=0 .
$$

Substituting with $I=e^{m z}$, then

$$
\left(c-\frac{D}{c}\right) m-\lambda+\exp (c T m)=0 .
$$

Denoting the left hand side of Eq. (19) by $f(m)$, then it is easy to see that $f^{\prime}(m)>0 \forall c \geq \sqrt{D}$ i.e. $f(m)$ is monotonically increasing. Furthermore $f(0)>0 \forall 0<\lambda<1$. Thus we have

Proposition 3: The solution $(S=1, I=0)$ for Eq. (18) subject to the boundary conditions (15) is asymptotically stable for $(z \rightarrow \infty)$, if the conditions (17) are satisfied. 
The derivation of the fraction of survivals $\sigma$ after the epidemic wave is similar to the case without delay and is given by Eq. (16).

Also, we will discuss one of the mechanisms of cross diffusion namely recruitment through signals [Saffre and Deneubourg 2002]. In this case swarming individuals e.g. insects recruit others via signals e.g. pheromone for ant colonies. Here we generalize the model of Saffre and Deneubourg to include diffusion, and study the boundedness and persistence of the solutions.

Let $u$ be the population density and $v$ be the pheromone density, then

$$
\begin{gathered}
\frac{\partial u}{\partial t}=(\alpha+\mu u) u(1-u)-\theta u v+D_{1} \frac{\partial^{2} u}{\partial x^{2}}, \\
\frac{\partial v}{\partial t}=\theta u v-v+D_{2} \frac{\partial^{2} v}{\partial x^{2}},
\end{gathered}
$$

where the parameters $\alpha, \beta, \theta, \mu$ are nonnegative. There are three homogeneous equilibrium solutions

$$
(u, v)=(0,0),(1,0),\left(\frac{1}{\theta}, \frac{1}{\theta^{3}(\theta-1)(\alpha \theta+\mu)}\right) .
$$

The solution $(0,0)$ is unstable, the solution $(1,0)$ is asymptotically stable if $\theta<1$. The third (positive) solution is asymptotically stable if

$$
\theta>1, \mu(1-2 . \theta)<\alpha .
$$

The results of Cantrell et al [Cantrell et al 1993] are directly applicable to the system (20), hence the solutions of Eq. (20) are persistent if

$$
\alpha>D_{1}\left(\frac{\pi}{L}\right)^{2}, \quad \theta>1+D_{2}\left(\frac{\pi}{L}\right)^{2} .
$$

To study the boundedness of the solutions to Eq. (20), we will consider only solutions satisfying the following condition

$$
u(0) \geq 0, v(0) \geq 0 \Rightarrow u(t) \geq 0, v(t) \geq 0 \forall t>0 .
$$

It is direct to see that $\partial u / \partial t<0 \forall u>1$, hence sup $u(t) \leq 1$. Consequently $\partial v / \partial t \leq(\theta-1) v \Rightarrow \forall \theta \leq 1$, then $v(t) \leq v(0)$. Thus we have:

\section{Proposition 4:}

(i) For $0 \leq \theta \leq 1$, the solutions of the system (20) with $D_{1}=D_{2}=0$ are bounded in $R_{+}^{2}=\{(x, y)$ such that $x \geq 0, y \geq 0\}$. 
(ii) The solutions of Eq. (20) are persistent given that the conditions (21) are valid.

\section{Cross diffusion and evolutionarily stable strat- egy}

Evolutionarily stable strategy ESS [Hofbauer and Sigmund 1998] is an important concept in population dynamics. Consider a population in which each individual adopts one of $k$ possible strategies. A strategy $I^{*}$ is ESS if for all $I \neq I^{*}$ (yet close to it), then

(i) $A\left(I^{*}, I^{*}\right)>A\left(I, I^{*}\right)$; or

(ii) If $A\left(I^{*}, I^{*}\right)=A\left(I, I^{*}\right)$, then $A\left(I^{*}, I\right)>A(I, I)$,

where $A$ is the payoff matrix. The dynamics of ESS is given by replicator dynamics

$$
\frac{\mathrm{d} p_{i}}{\mathrm{~d} t}=p_{i}\left[(A p)_{i}-p A p\right], i=1,2,3, \ldots, k,
$$

where $p_{i}$ is the fraction of the population adopting the strategy $i$.

Recently Hofbauer has introduced the concept of spatial game [Hofbauer 1999], where the system is given by

$$
\frac{\partial p_{i}}{\partial t}=p_{i}\left[(A p)_{i}-p A p\right]+D_{i} \frac{\partial^{2} p_{i}}{\partial x^{2}}
$$

This concept is generalized to asymmetric games [Ahmed et al. 2002]. Here we generalize it further to contain cross diffusion in addition to ordinary diffusion. The equations become

$$
\frac{\partial p_{i}}{\partial t}=p_{i}\left[(A p)_{i}-p A p\right]+D_{i} \frac{\partial^{2} p_{i}}{\partial x^{2}}+\sum_{j \neq i}^{k} D_{i j} \frac{\partial^{2} p_{j}}{\partial x^{2}}
$$

The condition $\sum_{i} p_{i}=1$ implies $D_{i}-D_{k}+\sum_{j \neq i}^{k} D_{i j}=0$.

Applying to the hawk(H)-dove(D)- retaliate(R) game [Maynard Smith 1982] where players either fight for a given prize with value $v$ or agree to share it. The first strategy is called hawk $(\mathrm{H})$ and its drawback is that fight 
costs value $c>v$. The second strategy is called dove (D) and its drawback is that it loses against hawks. The third strategy is called retaliate where the player imitates the strategy of the opponent. Hence he (or she) is a hawk (dove) if the opponent is a hawk (dove). Thus the payoff matrix $A$ is

$$
A=\left[\begin{array}{ccc}
\frac{1}{2}(v-c) & v & \frac{1}{2}(v-c) \\
0 & \frac{v}{2} & \frac{v}{2} \\
\frac{1}{2}(v-c) & \frac{v}{2} & \frac{v}{2}
\end{array}\right]
$$

where $c$ and $v$ are constants and $c>v>0$. Let $p_{1}\left(p_{2}\right)$ be the fraction of population adopting $\mathrm{H}(\mathrm{D})$ strategy, respectively, then the system (24) becomes

$$
\begin{aligned}
\frac{\partial p_{1}}{\partial t}= & p_{1}\left[-\frac{c}{2}+\frac{p_{1}}{2\left(c-\frac{v}{2}\right)}+\frac{p_{2}(v+c)}{2}-\frac{p_{1}^{2} c}{2}-c p_{1} p_{2}\right]+ \\
& D_{1} \frac{\partial^{2} p_{1}}{\partial x^{2}}+D_{12} \frac{\partial^{2} p_{2}}{\partial x^{2}} \\
\frac{\partial p_{2}}{\partial t}= & p_{2}\left[p_{1}\left(c-\frac{v}{2}\right)-\frac{p_{1}^{2} c}{2}-c p_{1} p_{2}\right]+D_{2} \frac{\partial^{2} p_{2}}{\partial x^{2}}+D_{21} \frac{\partial^{2} p_{1}}{\partial x^{2}}
\end{aligned}
$$

Assuming $1 \geq x \geq 0$, then linear stability analysis shows that $p_{1}=p_{2}=0$ is unstable if

$$
D_{12} D_{21}>D_{2}\left(D_{1}+\frac{c}{2 \pi^{2}}\right) .
$$

Thus cross diffusion can destabilize equilibrium solution, in some cases, if $D_{12}, D_{21}$ are large enough.

\section{Cross diffusion and the dynamics of learn- ing in multiagent systems}

Recently [Sato and Crutchfield 2002] have studied the dynamics of learning in multiagent systems, where the agents use reinforcement learning. They showed that, although the agents are not directly interacting with each other, a collective game between them arises through their interaction with the environment. Such interactions can be modelled via replicator type equations.

Consider two agents $u$ and $v$ with $n$ possible actions, let $u_{j}(t)\left(v_{j}(t)\right)$ be a measure of the probability that the agent $u(v)$ will take the action $j$ at 
time $t$. Sato and Crutchfield [Sato and Crutchfield 2002] have shown that $u_{j}(t), v_{j}(t)$ satisfy the following equations

$$
\begin{aligned}
\frac{\mathrm{d} u_{i}}{\mathrm{~d} t} & =u_{i} \beta_{1}\left[(A v)_{i}-u A v\right]+\alpha_{1} u_{i} \sum_{j} u_{j} \ln \left(\frac{u_{j}}{u_{i}}\right), \\
\frac{\mathrm{d} v_{i}}{\mathrm{~d} t} & =v_{i} \beta_{2}\left[(B u)_{i}-v B u\right]+\alpha_{2} v_{i} \sum_{j} v_{j} \ln \left(\frac{v_{j}}{v_{i}}\right),
\end{aligned}
$$

where $A$ and $B$ are payoff (reward) matrices for $u$ and $v$, respectively, and $\alpha_{1}, \alpha_{2}, \beta_{1}, \beta_{2}$ are nonnegative constants. If $\alpha_{1}=\alpha_{2}=0$, then one regains the standard asymmetric replicator equations [Hofbauer and Sigmund 1998, Ahmed et al. 2002]. For simplicity we consider the following payoff matrices $A=\operatorname{diag}\left(a_{1}, a_{2}\right), B=\operatorname{diag}\left(b_{1}, b_{2}\right), a_{i} \geq 0, b_{i} \geq 0$, hence the system $(27)$ becomes $\left(u_{1}=u, u_{2}=1-u, v_{1}=v, v_{2}=1-v\right)$

$$
\begin{aligned}
& \frac{\mathrm{d} u}{\mathrm{~d} t}=\beta_{1} u(1-v)\left(u\left(a_{1}+a_{2}\right)-a_{2}\right), \\
& \frac{\mathrm{d} v}{\mathrm{~d} t}=\beta_{2} v(1-u)\left(v\left(b_{1}+b_{2}\right)-b_{2}\right) .
\end{aligned}
$$

There are four equilibria $(u, v): E_{0}=(0,0), E_{1}=\left(a_{2} /\left(a_{1}+a_{2}\right), 0\right), E_{2}=$ $\left(0, b_{2} /\left(b_{1}+b_{2}\right)\right), E_{3}=(1,1)$. The solution $E_{0}$ is asymptotically stable while $E_{1}, E_{2}, E_{3}$ are saddle points, hence

Corollary 1: The system (28) is not persistent.

Including cross diffusion the system becomes

$$
\begin{aligned}
\frac{\mathrm{d} u}{\mathrm{~d} t} & =\beta_{1} u(1-v)\left(u\left(a_{1}+a_{2}\right)-a_{2}\right)+D_{12} \frac{\partial^{2} v}{\partial x^{2}} \\
\frac{\mathrm{d} v}{\mathrm{~d} t} & =\beta_{2} v(1-u)\left(v\left(b_{1}+b_{2}\right)-b_{2}\right)+D_{21} \frac{\partial^{2} u}{\partial x^{2}}
\end{aligned}
$$

where $1 \geq x \geq 0$, and the following Dirichlet boundary conditions are assumed $u(0, t)=u(1, t)=v(0,1)=v(1, t)=0$. Assuming

$$
u=u_{e q}+u_{0} \exp (\sigma t) \sin \pi x, v=v_{e q}+v_{0} \exp (\sigma t) \sin \pi x,
$$

where $\left(u_{e q}, v_{e q}\right)$ is one of the equilibrium solutions $E_{1}, E_{2}, E_{0}$, one gets 
Proposition 5: The following condition is necessary for persistence of the system (29):

$$
D_{12} D_{21}>\max \left\{\frac{\beta_{1} \beta_{2} a_{2} b_{2}}{\pi^{4}}, \frac{\beta_{1} \beta_{2} a_{1} b_{2}}{\pi^{4}\left(a_{1}+a_{2}\right)}, \frac{\beta_{1} \beta_{2} b_{1} a_{2}}{\pi^{4}\left(b_{1}+b_{2}\right)}\right\} .
$$

Proof. The condition (30) implies that $E_{1}, E_{2}, E_{0}$ are unstable which is a necessary condition for the persistence of the system.

\section{Conclusions}

Conditions for Turing instability and persistence in the presence of cross diffusion are derived. The predator-prey model is generalized to the cross diffusion case. Several types of interactions are assumed. .

Stability and persistence of the solutions of an epidemic model with cross diffusion is studied.

The effect of cross diffusion to evolutionary games is studied. In some cases, cross diffusion destabilizes the equilibrium solution.

Conditions for persistence and stability for the dynamics of learning in multiagent systems are concluded.

\section{Acknowledgments:}

We thank R. S. Cantrell and C. Cosner for their comments. Also we thank the referees for their helpful comments.

\section{References}

Ahmed E., Hegazi A. S. and Elgazzar A. S. (2002), On spatial asymmetric games. Advances in complex systems 5, 433-444.

Cantrell R. S., Cosner C. and Hutson V. (1993), Permanence in ecological systems with spatial heterogeneity. Proceeding of the Royal. Society of Edinburgh 123A, 533-559. 
Chattopadhyay J. and Chatterjee S. (2001), Cross diffusional effects in a Lotka-Volterra competitive system, Nonlin. Phen. in Complex Sys. 4, 364-369.

Chen L., Hsiao L. and Li Y.(2003), Strong solution to a kind of cross diffusion parabolic system, Comm. Math. Sci. 1, 799.

Hofbauer J. (1999), The spatially dominant equilibrium of a game. Annals of Operations Research 89, 233-251.

Hofbauer J. and Sigmund K. (1998), Evolutionary games and population dynamics. Cambridge university press, Cambridge.

Le D. (2003), Cross diffusion systems on n-dimensional domains, Electronic J. Diff. Eqns, Conference 10, 193.

Maynard Smith J. (1982), Evolution and theory of games, Cambridge Univ. Press U.K.

Mendez V. (1998), Epidemic model with infected infectious period. Phys. Rev. E 57, 3622-3624.

Murray J.D.. (2003), "Mathematical biology", Springer Publishers..

Okubo A. (1980), Diffusion and ecological problem. Springer-Verlag, Berlin..

Saffre F. and Deneubourg J. L. (2002), Swarming strategies for cooperative species. J. Theor. Biol. 214, 441-451.

Sato Y. and Crutchfield J. P. (2002), Coupled replicator equations for the dynamics of learning in multiagent systems, Nlin AO/0204057. 